\title{
The Central Bright Spot Sign: A Potential New MR Imaging Sign for the Early Diagnosis of Anterior Ischemic Optic Neuropathy due to Giant Cell Arteritis
}

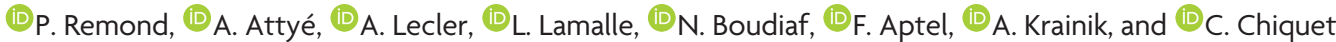

\begin{abstract}
BACKGROUND AND PURPOSE: A rapid identification of the etiology of anterior ischemic optic neuropathy is crucial because it determines therapeutic management. Our aim was to assess MR imaging to study the optic nerve head in patients referred with anterior ischemic optic neuropathy, due to either giant cell arteritis or the nonarteritic form of the disease, compared with healthy subjects.
\end{abstract}

MATERIALS AND METHODS: Fifteen patients with giant cell arteritis-related anterior ischemic optic neuropathy and 15 patients with nonarteritic anterior ischemic optic neuropathy from 2 medical centers were prospectively included in our study between August 2015 and May 2016. Fifteen healthy subjects and patients had undergone contrast-enhanced, flow-compensated, 3D T1-weighted MR imaging. The bright spot sign was defined as optic nerve head enhancement with a 3-grade ranking system. Two radiologists and 1 ophthalmologist independently performed blinded evaluations of MR imaging sequences with this scale. Statistical analysis included interobserver agreement.

RESULTS: MR imaging scores were significantly higher in patients with giant cell arteritis-related anterior ischemic optic neuropathy than in patients with nonarteritic anterior ischemic optic neuropathy $(P \leq .05)$. All patients with giant cell arteritis-related anterior ischemic optic neuropathy (15/15) and 7/15 patients with nonarteritic anterior ischemic optic neuropathy presented with the bright spot sign. No healthy subjects exhibited enhancement of the anterior part of the optic nerve. There was a significant relationship between the side of the bright spot and the side of the anterior ischemic optic neuropathy $(P \leq .001)$. Interreader agreement was good for observers ( $\kappa=0.815)$.

CONCLUSIONS: Here, we provide evidence of a new MR imaging sign that identifies the acute stage of giant cell arteritis-related anterior ischemic optic neuropathy; patients without this central bright spot sign always had a nonarteritic pathophysiology and therefore did not require emergency corticosteroid therapy.

ABBREVIATIONS: $\mathrm{AION}=$ anterior ischemic optic neuropathy; $\mathrm{FFE}=$ fast-field echo; GCA = giant cell arteritis; GCA-AION = giant cell arteritis-related anterior ischemic optic neuropathy; NA-AION = nonarteritic anterior ischemic optic neuropathy

A nterior ischemic optic neuropathy (AION) is the most common acute optic neuropathy in individuals older than 50 years of age, with an incidence of $2-10$ per 100,000 people per year. ${ }^{1,2}$ AION results from ischemic events within the optic nerve; these may be related to systemic vasculitis affecting large- and medium-caliber vessels in the arteritic form, due to giant cell arteritis (GCA-AION) or to blood flow disturbances associated with

Received November 3, 2016; accepted after revision February 25, 2017.

From the Department of Neuroradiology and MRI (P.R., A.A., A.K.), SFR RMN Neurosciences, and Department of Ophthalmology (P.R., F.A., C.C.), University Hospital of Grenoble, Grenoble, France; University Grenoble Alpes (A.A., L.L., N.B., A.K.), IRMaGe, Grenoble, France; and Department of Neuroradiology (A.L.), Rothschild Foundation, Paris, France.

The study was supported by Guerbet SA for MRI in Healthy Subjects.

Please address correspondence to Arnaud Attyé, MD, Neuroradiology and MR Unit, CS 10217, Grenoble University Hospital, F-38043, Grenoble Cedex 9, France; e-mail: aattye@chu-grenoble.fr

http://dx.doi.org/10.3174/ajnr.A5205 nonarteritic ischemic optic neuropathy (NA-AION). A rapid identification of the etiology is crucial because it determines the therapeutic management; corticosteroids must be promptly administered in cases of GCA-AION. Establishing the diagnosis of giant cell arteritis (GCA) may be challenging. The histopathologic result of a temporal artery biopsy has been considered the diagnostic standard but should not delay the prompt institution of steroid therapy. Some clinical signs suggest an arteritic etiology, including amaurosis fugax and systemic symptoms; a marked deterioration of visual acuity; more extensive visual field defects; diplopia; and the findings of fundus examination and fluorescein or indocyanine green angiography. ${ }^{3}$ However, ophthalmologists require a rapid diagnostic test to definitively rule out this diagnosis in an emergency setting. In GCA-AION, the granulomatous inflammatory artery infiltrate consists of T-lymphocytes, macrophages, and multinucleated giant cells. The pathogenesis is still not fully understood, though there have been major advances in 
recent years. Cytotoxic mediators and growth factors trigger the remodeling of the arterial wall, leading to ischemic manifestations by luminal stenosis. ${ }^{4}$

An MR imaging examination is often used to narrow the differential diagnosis of optic disc edema and to highlight retrobulbar optic nerve enhancement in optic neuritis, a differential consideration of AION. ${ }^{5,6}$ In addition, imaging of branches of the external carotid artery may be useful in the diagnosis of GCA. ${ }^{7}$ In contrast, radiologic data have traditionally failed to obtain positive signs for diagnosing AION; the most common finding in these patients is brain leukoaraiosis, particularly in patients with the nonarteritic form of the disease. ${ }^{8}$ MR imaging is the technique of choice for studying subtle ischemic events, either with contrast media or diffusion imaging. However, diffusion-weighted MR imaging is prone to distortion and motion artifacts, which may be problematic for the study of the anterior optic pathway.

We raised the hypothesis that cytotoxic ischemia is more pronounced in GCA-AION compared with NA-AION, potentially leading to more permeable vessels in the former. We further hypothesized that damage to the anterior optic nerve microcirculation at the acute stage of AION might be revealed by contrastenhanced MR imaging through a focal enhancement of the optic nerve head, the "central bright spot sign."

\section{MATERIALS AND METHODS}

This was a multicenter, parallel-group imaging study that was registered with the ClinicalTrials.gov registry for healthy volunteers (NCT02529475). The study was approved by our institutional review board (6705/15-CHUG-02) for patients. It was conducted in accordance with the Declaration of Helsinki and international standards of Good Clinical Practice. Signed informed consent was obtained from all healthy volunteers.

\section{Study Population and Design}

Patients with typical MR imaging contraindications, such as implanted pacemakers, metallic foreign bodies, claustrophobia, contrast agent allergy, or renal dysfunction with a glomerular filtration rate of $<30 \mathrm{~mL} / \mathrm{h}$, were excluded.

Patients 18 years of age or older were consecutively included in this study if they presented with acute onset of AION symptoms between August 2015 and May 2016. A diagnosis of AION was made following a recent appearance of a painless decrease in visual acuity and/or visual field defects and a clinical examination for diffuse or sectoral optic disc edema and peripapillary hemorrhages. ${ }^{4}$ Fluorescein angiography (Heidelberg Engineering, Heidelberg, Germany) was performed to detect the early stages of choroidal ischemia, and a visual examination (Humphrey Automated Field Analyzer 30-2 SITA-standard; Zeiss, Dublin, California) was performed at admission.

These patients were consecutively included if they had undergone MR imaging with a 3D radiofrequency-spoiled T1weighted high-resolution, flow-compensated, fast-field echo (FFE) acquisition.

The diagnosis of GCA was based on being older than 50 years of age, systemic symptoms (anorexia, weight loss, jaw claudication, headache, scalp tenderness, abnormal temporal artery, neck pain, myalgia, malaise, and anemia), ophthalmologic examina-

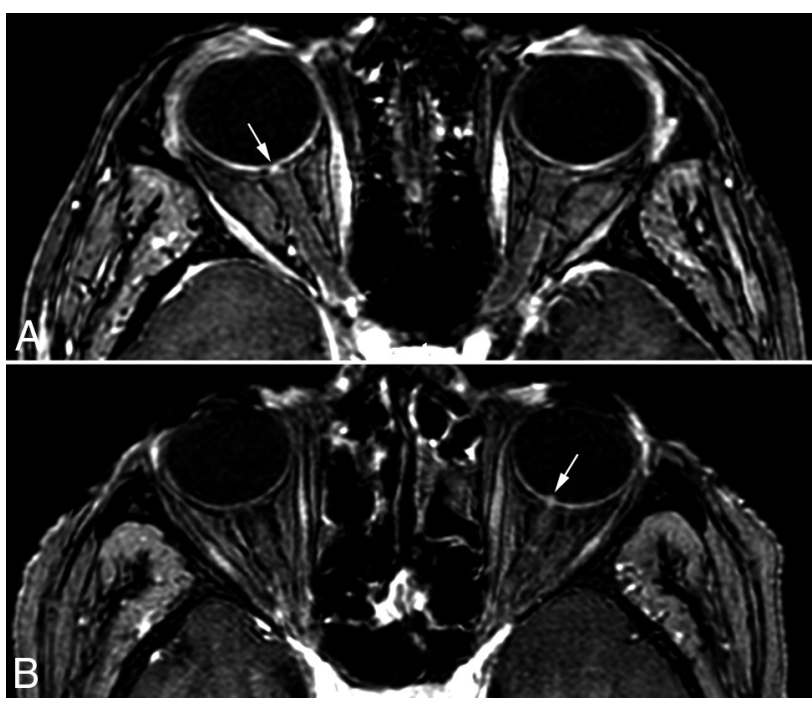

FIG 1. High-resolution $T 7$ sequences in the axial plane showing the bright spot sign ranking score. $A$, The first patient was referred with right nonarteritic AION and a score of 1, with no optic nerve head intravitreal protrusion but local enhancement (white arrow). $B$, The second patient was referred with left GCA-related AION and both protrusion and local enhancement, defined as score 2 (white arrow).

tion, biologic inflammatory syndrome with erythrocyte sedimentation, elevated C-reactive protein, and a biopsy of the temporal artery positive for GCA.

Healthy volunteers were included in the control group and had undergone MR imaging with the same protocol, to ensure that normal vascularization of the optic nerve head could be distinguished from disease process.

\section{Imaging Diagnosis}

Two board-certified radiologists (A.A. and A.L.) and 1 ophthalmologist (P.R.) from 2 different university hospitals independently evaluated anonymized MR images of patients and healthy subjects. The readers were blinded to all clinical data. We performed the scans on a 3T MR imaging scanner, Achieva 3T TX (Philips Healthcare, Best, the Netherlands) with a 32-channel head coil. The MR imaging included a 3D radiofrequency-spoiled T1-weighted high-resolution, flow-compensated, FFE acquisition (magnitude image from a phase conventional angiography scan), by using the shortest TR and partial-echo TE (7.4 and 4.1 $\mathrm{ms}$, respectively), $12^{\circ}$ flip angle, $480-\mathrm{Hz} /$ pixel readout gradient, transverse orientation, 95 sections, voxel size acquisition $=0.6 \times$ $0.7 \times 0.8 \mathrm{~mm}^{3}$, voxel size reconstruction $=0.33,0.33$, and 0.40 $\mathrm{mm}^{3}$, right-left phase encoding, and scan time $=5$ minutes 19 seconds. This evaluation was added to T2 spin-echo with wholebrain coverage.

\section{MR Imaging Grading System}

For qualitative analyses, we visually evaluated the intensity of the optic nerve head with a 3-grade ranking system. Each optic nerve head was assigned a score as follows:

Score 0: no optic nerve head enhancement

Score 1: optic nerve head enhancement without intravitreal protrusion (Fig 1A)

Score 2: both optic nerve head enhancement and intravitreal protrusion (Fig 1B). 
Table 1: Number of patients with the bright spot sign for each reader

\begin{tabular}{cccc}
\hline Reader & $\begin{array}{c}\text { Healthy } \\
\text { Subjects } \\
(\boldsymbol{n}=15)\end{array}$ & $\begin{array}{c}\text { NA-AION } \\
(\boldsymbol{n}=15)\end{array}$ & $\begin{array}{c}\text { GCA-AION } \\
(\boldsymbol{n}=15)\end{array}$ \\
\hline Reader 1 & $0 / 15$ & $7 / 15$ & $15 / 15$ \\
Reader 2 & $0 / 15$ & $6 / 15$ & $15 / 15$ \\
Reader 3 & $0 / 15$ & $6 / 15$ & $15 / 15$ \\
\hline
\end{tabular}

In this study, score 0 represented physiologic features, and scores 1 and 2 represented active papillary disease.

Each observer rated the MR images of each patient as positive or negative for AION; for a positive MR imaging result, an optic nerve head score of 1 or 2 was required.

\section{Statistical Analysis}

Data were analyzed with SPSS software, Version 22.0 (IBM, Armonk, New York). Continuous data are presented as means with SDs. Age differences between healthy participants and patients were tested by using the Student $t$ test. Categoric data were reported as frequency and percentages. The Pearson $\chi^{2}$ test was used for the independence of the side of the central bright spot sign and the clinical side of the disease in the patient group. Sensitivity, specificity, positive/negative predictive values, and positive/negative likelihood ratios of the MR imaging examinations were calculated for each observer. The interobserver agreement was calculated by using the Fleiss $\kappa$ test; a $\kappa$ value $>0.80$ represented outstanding agreement. We set the significance threshold ( $P$ values) at $.05 .^{9}$

\section{RESULTS}

Fifteen healthy volunteers ( 8 women, 7 men) were included in this study, with a mean age of $62 \pm 6$ years. Thirty patients ( 8 women, 22 men) were included with a mean age of $71 \pm 11$ years. Fifteen patients presented with NA-AION, while 15 presented with GCAAION, confirmed with histopathologic examination. The delay between ocular symptoms and MR imaging was $3 \pm 1.2$ days. No adverse effects were observed after injection of gadoteric acid. There was no significant difference in the ages of healthy and patient groups.

\section{Bright Spot Findings}

With contrast-enhanced MR imaging, patients in the control group exhibited no enhancement of the optic nerve head. All patients with GCA-AION and 7 of 15 patients (46\%) with NAAION presented with the bright spot sign, a local enhancement of the optic nerve head on the high-resolution T1 sequence. The interobserver agreement for the 3 -grade ranking system was high $(\kappa=0.82)$ (Table 1$)$.

We found a significant relationship between the side of the bright spot and the side of clinical disease $(P \leq .001)$. Table 2 summarizes the sensitivity, specificity, positive/negative predictive values, and positive/negative likelihood ratios of the MR imaging examinations.

MR imaging allows to distinguish GCA-AION from NAAION with sensitivity of $100 \%$, and specificity ranging from $53.3 \%$ to $60 \%$ for observer. Most important, all patients with AION and without a bright spot sign were diagnosed with NA-
AION (Table 2). This finding was supported by a negative likelihood ratio of $<0.1$ (ie, very useful for identifying patients who did not have GCA-AION).

In patients with NA-AION, MR imaging had a moderate sensitivity and high specificity and positive predictive value, compared with healthy volunteers (Table 2).

In patients with GCA-AION versus healthy subjects, the sensitivity, specificity, and negative and positive predictive values of MR imaging were $100 \%$ for each reader because the central bright spot sign was observed in all these patients.

The presence of the central bright spot sign was not significantly associated with age in either group.

\section{DISCUSSION}

Here, we have demonstrated the early presence of a new MR imaging sign in the acute phase of AION. All patients with GCAAION exhibited optic nerve head enhancement, compared with only $50 \%$ of patients with NA-AION $(P \leq .05)$ and $0 \%$ of healthy volunteers $(P \leq .01)$. Therefore, in our series, the absence of optic nerve head enhancement in patients with AION precluded a diagnosis of giant cell arteritis.

In the clinical management of patients with AION, it is crucial to diagnose GCA promptly because early treatment with corticosteroids decreases both the risk of contralateral eye injury and recurrence on the original side ${ }^{1,3}$ and also decreases the risk of systemic complications. To date, there is limited evidence available regarding the value of MR imaging for patients with ischemic optic neuropathy. In a previous study with T1 inversion recovery-weighted imaging, Rizzo et $\mathrm{al}^{4}$ observed abnormalities in the clinically affected optic nerve of patients with optic neuritis (ie, associated with demyelinating brain disorders), whereas no abnormal signal was detected in patients with AION. Recently, an MR imaging study of 3 patients with AION highlighted possible optic nerve perineural sheath and optic chiasma enhancement. ${ }^{10}$ However, the optic nerve head was not studied in these patients.

This is the first MR imaging study that demonstrates an optic nerve head morphologic sign in patients with AION, to our knowledge. The negative predictive value of this sign strongly suggests that the absence of the bright spot sign rules out a diagnosis of GCA-AION.

In addition to morphologic brain examination, it was recently proposed that diffusion-weighted MR imaging and tractography postprocessing may help diagnose AION at the subacute stage. ${ }^{11}$ Focusing on diffusion-weighted MR imaging, Bender et $\mathrm{al}^{12}$ described 5 cases of acute optic ischemia with diffusion restriction and reduced apparent diffusion coefficient, while Wan et $\mathrm{al}^{11}$ found only 2 cases of AION in a cohort of 42 patients. In a larger tractography study of 26 patients, Wang et $\mathrm{al}^{13}$ proposed several diffusion biomarkers in patients with AION that may serve as biomarkers of axonal and myelin damage in AION. However, diffusion-weighted MR imaging is prone to distortion and motion artifacts. This may be problematic for the study of optic pathways and extraction of quantitative values. Furthermore, the tensor model typically used for tractography in this area is particularly challenging to apply to the low signal-to-noise ratio areas. ${ }^{14}$ In addition, diffusion-weighted MR imaging depends on axonal 
Table 2: Sensitivity, specificity, positive/negative predictive values, and positive/negative likelihood ratios of the MR imaging bright spot sign in healthy subjects and patients with GCA-AION or NA-AION

\begin{tabular}{|c|c|c|c|c|c|c|c|c|c|c|c|}
\hline & $\begin{array}{c}\text { No. of } \\
\text { Participants }\end{array}$ & $\begin{array}{c}\text { TP } \\
\text { Results }\end{array}$ & $\begin{array}{c}\text { TN } \\
\text { Results }\end{array}$ & $\begin{array}{c}\text { FP } \\
\text { Results }\end{array}$ & $\begin{array}{c}\text { FN } \\
\text { Results }\end{array}$ & $\begin{array}{c}\text { Sensitivity } \\
(\%)\end{array}$ & $\begin{array}{c}\text { Specificity } \\
(\%)\end{array}$ & $\begin{array}{l}\text { PPV } \\
(\%)\end{array}$ & $\begin{array}{l}\text { NPV } \\
(\%)\end{array}$ & PLR & NLR \\
\hline \multicolumn{12}{|c|}{ NA-AION vs GCA-AION } \\
\hline Observer 1 & 30 & 15 & 8 & 7 & 0 & 100 & 53.3 & 68.2 & 100 & 2.1 & 0 \\
\hline Observer 2 & 30 & 15 & 9 & 6 & 0 & 100 & 60 & 71.4 & 100 & 3.5 & 0 \\
\hline Observer 3 & 30 & 15 & 9 & 6 & 0 & 100 & 60 & 71.4 & 100 & 3.5 & 0 \\
\hline \multicolumn{12}{|c|}{ NA-AION vs healthy subjects } \\
\hline Observer 1 & 30 & 7 & 15 & 0 & 8 & 46.7 & 100 & 100 & 65.2 & & 0.5 \\
\hline Observer 2 & 30 & 6 & 15 & 0 & 9 & 40 & 100 & 100 & 62.5 & & 0.6 \\
\hline Observer 3 & 30 & 6 & 15 & 0 & 9 & 40 & 100 & 100 & 62.5 & & 0.6 \\
\hline \multicolumn{12}{|c|}{ GCA-AION vs healthy subjects } \\
\hline Observer 1 & 30 & 15 & 15 & 0 & 0 & 100 & 100 & 100 & 100 & & 0 \\
\hline Observer 2 & 30 & 15 & 15 & 0 & 0 & 100 & 100 & 100 & 100 & & 0 \\
\hline Observer 3 & 30 & 15 & 15 & 0 & 0 & 100 & 100 & 100 & 100 & & 0 \\
\hline
\end{tabular}

Note:-FN indicates false-negative; FP, false-positive; PLR, positive likelihood ratio; NLR, negative likelihood ratio; NPV, negative predictive value; PPV, positive predictive value; TN, true-negative; TP, true-positive.

integrity but also on other parameters such as axon diameter and the degree of myelination or the space between axons. ${ }^{15}$

A published case report of a patient with NA-AION has previously described this marked enhancement of the optic disc, similar to the bright spot sign. ${ }^{16}$ Friedland et al ${ }^{17}$ reported that this phenomenon could be associated with increased perfusion, a reparative reaction to ischemia. Hayreh ${ }^{18}$ determined that optic disc edema is always present in acute AION, due to 2 factors: an axonal ischemia mechanism with capillary leakage in the optic nerve head and a venous stasis secondary to the acute stroke. Optic nerve head enhancement on MR imaging assessment has previously been described in patients with pseudotumor cerebri compared with controls ${ }^{19}$ and in 1 patient at the acute stage of Leber hereditary optic neuropathy. ${ }^{20}$

In our study, the bright spot sign was consistently observed in patients with GCA-AION and is concordant with the cytotoxic ischemia of this disease. ${ }^{21}$

We acknowledge that there are several limitations of this study. First, we should acknowledge a small sample size for patients with AION. While these results are encouraging, further multicenter studies that involve a large number of patients with AION, which is a rare disease in the context of GCA, should be performed to validate the early use of enhanced and high-resolution MR images.

In addition, we performed the scanning at the subacute phase with a mean delay of $3 \pm 1.2$ days. In the absence of other time points, however, we cannot currently determine the optimal delay for the examination, especially relating to the hours immediately after the clinical event. Follow-up studies would also be of interest to observe the evolution of the MR imaging signal with the time, with the potential limit of repeated injections of gadolinium which may be associated with contrast media accumulation in the brain parenchyma of healthy subjects. ${ }^{22,23}$ Here, we used a macrocyclic gadolinium chelate administration that, to date, has not been associated with the presence of hypersignal intensity in the dentate nucleus. ${ }^{23}$

A long-term, clinical follow-up of patients would allow determining whether there is a correlation among the central bright spot sign, visual field defects, and/or involvement of the retinal nerve fiber layer of the optic nerve and macular ganglion cells, as has previously been demonstrated with optical coherence tomog- raphy. ${ }^{24}$ It might also reveal a possible predictive power for the likelihood of recovery or visual prognosis according to the absence or presence of the signal and its visual scale ranking.

\section{CONCLUSIONS}

This preliminary study suggests that patients with suspected ischemic optic neuropathy can potentially benefit from MR imaging in the emergency setting, to differentiate GCA-AION and NAAION and definitively exclude the arteritic form.

\section{ACKNOWLEDGMENTS}

The authors acknowledge the valuable assistance of Patrice Jousse for editing the MR imaging pictures and drawings. We also thank Dr Louise Ball for critically editing the manuscript.

Disclosures: Arnaud Attyé-RELATED: Grant: Guerbet SA, Comments: The MRI for Healthy Subjects was funded by Guerbet SA*. Christophe Chiquet-UNRELATED: Board Membership: Allergan, Thea, ABBVIE. *Money paid to the institution.

\section{REFERENCES}

1. Johnson LN, Arnold AC. Incidence of nonarteritic and arteritic anterior ischemic optic neuropathy: population-based study in the state of Missouri and Los Angeles County, California. J Neuroophthalmol 1994;14:38-44 Medline

2. Hattenhauer MG, Leavitt JA, Hodge DO, et al. Incidence of nonarteritic anterior ischemic optic neuropathy. Am J Ophthalmol 1997; 123:103-07 CrossRef Medline

3. Hayreh SS, Podhajsky PA, Zimmerman B. Occult giant cell arteritis: ocular manifestations. Am J Ophthalmol 1998;125:521-26 CrossRef Medline

4. Rizzo JF 3rd, Andreoli CM, Rabinov JD. Use of magnetic resonance imaging to differentiate optic neuritis and nonarteritic anterior ischemic optic neuropathy. Ophthalmology 2002;109:1679-84 CrossRef Medline

5. Hoorbakht H, Bagherkashi F. Optic neuritis, its differential diagnosis and management. Open Ophthalmol J 2012;6:65-72 CrossRef Medline

6. Klink T, Geiger J, Both M, et al. Giant cell arteritis: diagnostic accuracy of MR imaging of superficial cranial arteries in initial diagnosis-results from a multicenter trial. Radiology 2014;273:844-52 CrossRef Medline

7. Argyropoulou MI, Zikou AK, Tzovara I, et al. Non-arteritic anterior ischaemic optic neuropathy: evaluation of the brain and optic pathway by conventional MRI and magnetisation transfer imaging. Eur Radiol 2007;17:1669-74 CrossRef Medline 
8. Hayreh SS. Ischemic optic neuropathy. Prog Retin Eye Res 2009;28: 34-62 CrossRef Medline

9. Landis JR, Koch GG. The measurement of observer agreement for categorical data. Biometrics 1977;33:159-74 CrossRef Medline

10. D'Souza NM, Morgan ML, Almarzouqi SJ, et al. Magnetic resonance imaging findings in giant cell arteritis. Eye (Lond) 2016;30:758-62 CrossRef Medline

11. Wan $\mathrm{H}$, Sha $\mathrm{Y}$, Zhang F, et al. Diffusion-weighted imaging using readout-segmented echo-planar imaging, parallel imaging, and two-dimensional navigator-based reacquisition in detecting acute optic neuritis. J Magn Reson Imaging 2016;43:655-60 CrossRef Medline

12. Bender B, Heine C, Danz S, et al. Diffusion restriction of the optic nerve in patients with acute visual deficit. J Magn Reson Imaging 2014;40:334-40 CrossRef Medline

13. Wang MY, Qi PH, Shi DP. Diffusion tensor imaging of the optic nerve in subacute anterior ischemic optic neuropathy at $3 \mathrm{~T}$. AJNR Am J Neuroradiol 2011;32:1188-94 CrossRef Medline

14. Farquharson S, Tournier JD, Calamante F, et al. White matter fiber tractography: why we need to move beyond DTI. J Neurosurg 2013; 118:1367-77 CrossRef Medline

15. Beaulieu C, De Crespigny A, Tong DC, et al. Longitudinal magnetic resonance imaging study of perfusion and diffusion in stroke: evolution of lesion volume and correlation with clinical outcome. Ann Neurol 1999;46:568-78 Medline

16. Yovel OS, Katz M, Leiba H. Magnetic resonance imaging of luxury perfusion of the optic nerve head in anterior ischemic optic neuropathy. J Neuroophthalmol 2012;32:256-58 CrossRef Medline
17. Friedland S, Winterkorn JM, Burde RM. Luxury perfusion following anterior ischemic optic neuropathy. J Neuroophthalmol 1996;16: 163-71 CrossRef Medline

18. Hayreh SS. Fluids in the anterior part of the optic nerve in health and disease. Surv Ophthalmol 1978;23:1-25 CrossRef Medline

19. Brodsky MC, Vaphiades M. Magnetic resonance imaging in pseudotumor cerebri. Ophthalmology 1998;105:1686-93 CrossRef Medline

20. Lamirel C, Cassereau J, Cochereau I, et al. Papilloedema and MRI enhancement of the prechiasmal optic nerve at the acute stage of Leber hereditary optic neuropathy. J Neurol Neurosurg Psychiatry 2010;81:578-80 CrossRef Medline

21. Weyand CM, Goronzy JJ. Immune mechanisms in medium and large-vessel vasculitis. Nat Rev Rheumatol 2013;9:731-40 CrossRef Medline

22. Kanda $\mathrm{T}$, Ishii $\mathrm{K}$, Kawaguchi $\mathrm{H}$, et al. High signal intensity in the dentate nucleus and globus pallidus on unenhanced T1-weighted MR images: relationship with increasing cumulative dose of a gadolinium-based contrast material. Radiology 2014;270:834-41 CrossRef Medline

23. Kanda $\mathrm{T}$, Osawa $\mathrm{M}$, Oba $\mathrm{H}$, et al. High signal intensity in dentate nucleus on unenhanced T1-weighted MR images: association with linear versus macrocyclic gadolinium chelate administration. $R a$ diology 2015;275:803-09 CrossRef Medline

24. Goto K, Miki A, Araki S, et al. Time course of macular and peripapillary inner retinal thickness in non-arteritic anterior ischaemic optic neuropathy using spectral-domain optical coherence tomography. Neuroophthalmology 2016;40:74-85 CrossRef Medline 